Factors to Consider in Midwifery Care during Climacteric and Monopause Period

\author{
Bianca Locsin ${ }^{1}$, Maine Salvador ${ }^{1}$ \\ ${ }^{1}$ School of Midwifery, Manila Central University, Philippines \\ Received: Desembr 29, 2020 \\ Received in revised: January 14, 2021 \\ Accepted: January 28, 2021
}

\begin{abstract}
This study discusses the management of climacteric obstetrics and menopause. Menopause is the final feminine cycle or when the final monthly cycle happens, one of the mental viewpoints of changing self-concept amid menopause is unquestionably menopausal ladies ended up on edge around their bodies and frame self-concept approximately how their bodies are. The side effects experienced by ladies some time recently menopause cause the mother to be ill-equipped approximately physical and mental changes. To decrease this, ladies must get ready themselves both physically and mentally for menopause. Ladies who are going through menopause go through the primary stages counting premenopause, perimenopause, menopause, and postmenopause, and menopause for the most part happens in ladies matured 45-50 a long time.
\end{abstract}

Keywords: Midwifery, Climacteric, Monopause

\title{
Introduction
}

The climacterium is irresistible and a terrible picture for most women. Women who experience climax are described as sensitive, easy to cry, changeable emotions and feelings of being unattractive like their youth. The changes that occur in these women increasingly make women feel uncomfortable in the climacteric phase (Berterö, 2003).

Decreased activity of the ovaries with increasing age and changing levels of estrogen, progesterone and androgens affect sexual function in the climacteric period (Graziottin, \& Leiblum, 2005). Hormonal changes cause atrophy in the genital area, Vaginal atrophy causes dyspareunia, which can change a woman's approach to her partner or can decrease their sexual response to her partner (Levine et al., 2008).

Pruritus on the vulva, frequent urination, damage to the pelvic organs, stress, incontinence and constipation negatively affect sexual function and postmenopausal complaints indicate further development (Salvatore et al., 2015). In the climacteric period, there is decreased lubrication and libido, less orgasm in the sexual response cycle and sexual dysfunction (Fanfulla et al., 2013).

In the climacteric period, physiological changes, age-related difficulties and cultural characteristics affect the emotional balance of women. The vasomotor, cardiovascular, musculoskeletal, digestive, urogenital and mood changes with menopause also cause difficulties in sexual life (Monteleone et al., 2018).

Menopause is the last menstruation or when the last menstruation occurs, one of the psychological aspects of changing self-concept during menopause is definitely menopausal women become anxious about their bodies and form self-concept about how their bodies are. One of the psychological aspects of changing self-concept during menopause is definitely 
menopausal women becoming anxious about their bodies and forming a self-concept about how their bodies are doing, maybe they look in the mirror every day and sometimes every hour.

Menopause is physiological for every woman. Postmenopausal women will experience several complaints both physically and psychologically. The symptoms experienced by women before menopause cause the mother to be unprepared about physical and psychological changes. To reduce this, women must prepare themselves both physically and psychologically for menopause. Menopause is defined as the absence of menstrual periods for 12 months, but in general menopause can be said to be the end of the menstrual cycle in women.

\section{What is climacterium?}

Climacterium is an intermediate period between the reproductive period and the senium period. Usually this period is also called premenopause, between the ages of 40 years, characterized by irregular menstrual cycles, with prolonged and relatively heavy menstrual bleeding (Hale \& Burger, 2009).

Climacterium is a period that begins at the end of the reproductive stage, ends at the beginning of the senium and occurs in women aged 40-65 years. This period was marked by various kinds of complaints. Climacterium is not a pathological condition, but a normal transitional period that lasts several years before and after menopause. This is because the ovaries become old, so the hormone estrogen decreases and gonadotropin hormone increases (Eagleson et al., 2000)

The climacteric period is a period in which women adjust to decreased production of ovarian hormones that make a woman unable to reproduce. The climacteric age is also defined as the age of maturity where a person becomes more mature and wise both intellectually and emotionally. After a woman enters the climacteric period they will enter menopause.

Most women begin experiencing menopausal symptoms in their 40s and peak at age 50 (Thurston et al., 2017). The climacterium is irresistible and a terrible picture for most women. Women who experience climax are described as sensitive, easy to cry, changeable emotions and feelings of being unattractive like their youth. The changes that occur in these women increasingly make women feel uncomfortable in the climacteric phase (Berterö, 2003).

Decreased activity of the ovaries with increasing age and changing levels of estrogen, progesterone and androgens affect sexual function in the climacteric period (Graziottin, \& Leiblum, 2005). Hormonal changes cause atrophy in the genital area, Vaginal atrophy causes dyspareunia, which can change a woman's approach to her partner or can decrease their sexual response to her partner (Levine, Williams, \& Hartmann, 2008).

Pruritus on the vulva, frequent urination, damage to the pelvic organs, stress, incontinence and constipation negatively affect sexual function and postmenopausal complaints indicate further development (Pastore, Carter, Hulka, \& Wells, 2004). In the climacteric period, there is decreased lubrication and libido, less orgasm in the sexual response cycle and sexual dysfunction (Nappi et al., 2013).

In the climacteric period, physiological changes, age-related difficulties and cultural characteristics affect the emotional balance of women. The vasomotor, cardiovascular, musculoskeletal, digestive, urogenital and mood changes with menopause also cause difficulties in sexual life (Dennerstein et al., 2002) 
One of the factors for someone to be anxious in facing the climacteric period is knowledge. Broadly speaking, the period of a woman's life cycle goes beyond several stages including preconception, conception, pre-birth, pre-puberty, puberty, reproduction, climacteric and senium / elderly. Throughout the life cycle of a woman will experience a period that is physiological, as a phase where the aging process of women is marked by a shift from the reproductive period to the non-production period called the climacteric period (Wiknjosastro, 2002).

In women climacterium will experience aging ovaries, so they are unable to meet the hormone estrogen. The hormonal system throughout the body goes into decline which causes various physical and psychological changes (Manuaba, 2002).

The first complaints that are felt are vasomotor complaints (related to blood vessels) such as hot fishes (sudden hot flashes on the face, neck and chest), night sweats (excessive night sweats) and urogenital atrophy (thinning of the vaginal mucosa) which causing further consequences in the form of dryness of the vaginal canal so that during intercourse the husband and wife feel pain and decreased libido. Other complaints that are considered psychological and socio-cultural symptoms, for example, depression, headaches (Mulyani, 2013).

\section{Premenopause}

The period before the onset of peri menopause, that is, since the reproductive function begins to decline, until the onset of complaints or signs of menopause starting at the age of 40 years. Bleeding occurs due to decreased levels of estrogen, insufficiency of the corpus lutheum, failure of the ovulation process, so that menstrual abnormalities can manifest as amenorrhae, polimenorrhae and hypermenorrhae.

\section{Perimenopause}

The period with peak complaints, ranging from 1-2 years before and 1-2 years after menopause. The period of women experiencing the end of the arrival of menstruation until it stops completely. At this time menopause is still ongoing. Systematic complaints related to vasomotor, complaints that are often encountered are in the form of hot flushes, sweating profusely, depression, and feelings of irritability.

\section{Menopause}

After entering menopause, high FSH levels are always found (> $35 \mathrm{mIU} / \mathrm{ml}$ ). At the beginning of menopause sometimes low estrogen levels. In obese women, estrogen levels are usually high. If a woman has not had her period for 12 months and found FSH levels> $35 \mathrm{mIU} / \mathrm{ml}$ and estradiol levels $<30 \mathrm{pg} / \mathrm{ml}$, then the woman can be said to have experienced menopause.

\section{Post menopause}

The period after menopause until senile. The period lasts approximately 3-5 years after menopause. Local complaints in the lower urogenital system, atrophy of the vulva and vagina lead to reduced mucus production or painful intercourse. After the climacteric period is over, then women will experience a postmenopausal period, followed by a senile period. (Kasdu,2002).

Postmenopause is the phase in which the ovaries do not function at all. Estradiol levels are between 20-30 pg / ml, and gonadotropin hormone levels increase. Senium is the period after 
postmenopause, when a new balance has been reached in women's lives, so that there are no more vegetative or psychological disturbances.

\section{Early Signs of Climaterium And Menopause}

\section{Climatterium}

Signs and symptoms during the climacteric period are physical disorders such as menstrual abnormalities (prolonged menstrual cycles, irregular menstruation, heavy menstrual blood), constitutional complaints Palpitations, headaches (migraines), fatigue, joint muscle pain, back pain, weight gain .

Symptoms that arise include psychological symptoms in the form of lethargy, headache, dizziness, sleeplessness, gloomy feelings, irritability, decreased concentration of anxiety and depression. Hot flashes or feelings (hot flush) accompanied by profuse sweating. Heart palpitations. Difficulty taking a deep breath; Erratic appetite, often complaining of indigestion. Changes in menstrual patterns. Drying of the vagina and itching

Complaints of complaints of discomfort that arise in everyday life

Hot flushes are intense sensations of heat on the face and upper body (such as the neck and chest). With the touch of the hand you will feel an increase in temperature in the area. This symptom often occurs at night causing difficulty sleeping (Kasdu, 2002).

These episodes are described as a burning sensation that varies in frequency, duration, and severity, occasionally recurs, and usually lasts less than 5 minutes. This can be triggered by the environment, food, drink and stress (Nelson, 2008).

Excessive sweating is due to the radiating heat to the body due to the influence of hormones that regulate the body's thermostat at a lower temperature. As a result, the air temperature that was previously comfortable suddenly becomes too hot and the body starts to become hot, causing sweat to cool off.

Dry vagina due to changes in the reproductive organs, causing pain during intercourse. Unable to hold urine, namely when you get older, urine often cannot be held when you sneeze or cough. This is due to decreased estrogen so that one of the effects is urinary incontinence.

The loss of supporting tissue due to low levels of estrogen in the body affects the collagen network which functions as a supporting tissue for body tissues. Gaining weight when a woman turns 40 years old, usually her body easily gets fat, but on the other hand it is very difficult to lose it due to decreased estrogen and impaired exchange of basic substances for fat metabolism. Lack and loss of estrogen affects the production of the tear glands so that the eyes feel dry and itchy.

Bone and joint pain with increasing age means that some organs no longer undergo remodeling, including bones. Even experiencing a decline process due to the influence of changes in other organs (Kasdu, 2002).

\section{Menopause}

The symptoms of menopause are caused by changes in estrogen and progesterone levels. Because ovarian function is reduced, the ovaries produce less estrogen / progesterone and the body reacts. Some women experience only a few symptoms, while other women experience a variety of symptoms that range from mild to severe. This is normal. The gradual reduction in 
estrogen levels causes the body to slowly adjust to hormonal changes, but in some women this drop in estrogen levels occurs suddenly and causes severe symptoms. This often occurs when menopause is caused by removing the ovaries.

Hormonal changes in the body result in symptoms such as joint pain \& back pain, vaginal drying (resulting in pain during sexual intercourse), difficulty holding urine, high mood and emotional disturbances that cause stress, in addition to decreasing estrogen levels also result in tendency to increase blood pressure, gain weight \& increase cholesterol levels.

Some of the physical complaints that are signs and symptoms of menopause are irregular menstruation, hot flushes, insomnia, palpitations and weakness, sexual disturbances. Urinary tract symptoms such as painful urination, urinary tract infections and incontinence (Glasier \&Gebbie, 2005).

Some physical complaints are signs and symptoms of menopause, namely that every woman will begin to experience irregular menstrual cycles that can become longer or shorter until they finally stop. There is bleeding that comes irregular in a span of a few months and then stops completely. About $40 \%$ of women complain that their menstrual cycles are irregular. This situation increases to $60 \%$ in the 12 years before menstruation stops completely or menopause. This burning sensation is often accompanied by redness of the skin and sweating. Vaginal dryness occurs because the cervix secretes very little mucus. The cause is a lack of estrogen which causes the vaginal canal to become thinner, drier and less elastic. The genitals begin to shrink, the burrow is dry, causing pain during intercourse, holding back pee, especially when coughing, sneezing, laughing and orgasm.

Women experience a drop in their testosterone levels during pre menopause this can result in a loss of sexual desire. But for some women, libido problems are related to a lack of the hormone estrogen or the thinning of vaginal tissue (Baziad, 2003 ; Kasdu, 2002 ; Northrup, 2006 ; Wijayanti, 2009)

\section{Climacteric Disorders and Menopause}

According to Endang Purwoastuti, 2008, the symptoms and signs of climacteria are caused by changes in the reproductive organs, extragenital structure and the presence of clinical symptoms. In a failed ovary, the balance between the hormones estrogen and progesterone will be lost by decreasing hormone production, causing an effect on premenstrual syndrome and menstruation itself. Some women find that the syndrome worsens during the climacteric years and others experience it for the first time.

The uterus shrinks, caused by shrinking of the mucous membrane of the uterus (endometrial atrophy) is also caused by loss of fluid and changes in the shape of the connective tissue between cells. The uterine muscle fibers and vessels are thickened and protruding. After a woman is no longer menstruating, there is a thinning of the vaginal walls and vulvar tissue, the folds are reduced to watery secretions. frequent itching and pain during intercourse.

The spread of fat is found in the legs, lower abdomen and upper arms. About $20 \%$ of women climax experienced a marked increase. This is thought to have something to do with a decrease in estrogen and disruption of the basic substances of fat metabolism. Due to heat flare, there is an increase in blood pressure. In women aged 45-70 years, it is known that the increase in blood pressure begins during climacteria. Decrease or loss of estrogen levels leads to increased 
cholesterol and decreased total fat. The presence of hypertension and increased cholesterol levels lead to increased risk factors for atherosclerosis.

A sudden, hot feeling that starts from the upper part of the body and spreads to the face or the whole body. The next characteristic symptom is night sweats, which is kneeling with sweat when waking up at night, so it is necessary to change clothes at night followed by a cold feeling afterwards. As a result they are easily tired and easily irritated. This may be related to tensions due to night sweats, flushing, and other changes. Some women suffer from formication, which is an irritating sensation under the skin like the feeling of being bitten by an ant.

\section{Psychological Change}

In connection with physical changes there is also a shift or erosion in the personal psychic life concerned. These shifts and changes result in a crisis, and manifest themselves in psychological symptoms, including: depressions (moodiness), irritability, irritability, suspicion, anxiety, insomnia or sleeplessness due to confusion and confusion. restless.

\section{Etiology}

Before menstruation stops a woman, there are various changes and decreased function of the ovaries such as sclerosis of blood vessels, reduced number of follicles and decreased sex steroid synthesis due to decreased estrogen secretion, resulting in feedback disturbances in the pituitary (Hanifa, 1999).

Annual examinations of women who are at the climacteric period should include important matters such as height; women may lose $2.5 \mathrm{~cm} /$ more of TB. Skin; evaluation of integrity, wounds, and changes in the mole, mouth, teeth and gums. Pelvic exam; with attention to the changes that accompany the aging process. Rectum; check for masses and fissures.

\section{Organic Abnormalities During Menopause}

With constant stimulation of estrogen without interruption of progesterone, the opportunity for pathological states of the target organ of estrogen in the form of dysfunctional bleeding is increasing; there is a change in the genetalia apparatus into a benign tumor; uterine myoma, endometrial polyps, cervical polyps; uterine corpus carcinoma; breast malignancy.

\section{Treatment}

Not all postmenopausal women need to undergo hormone replacement therapy (TSH). Every woman should discuss the risks and benefits of TSH with her personal doctor. Many experts recommend TSH for the purpose of reducing unwanted menopausal symptoms; helps reduce vaginal dryness; prevent osteoporosis.

Some of the side effects of TSH include vaginal bleeding, breast tenderness, nausea, vomiting, flatulence, uterine cramps. To reduce the risk of TSH and still benefit from TSH, experts recommend adding progesterone to estrogen; adding testosterone to estrogen; using the lowest dose of estrogen; perform regular examinations, including pelvic examinations, and Pap smears so that abnormalities can be found as early as possible.

Midwifery Management Climacterium and Menopause

Management is a process in order to achieve goals by working together through people and other organizational resources. Midwifery care management is a problem-solving process used as a 
method for organizing thoughts or actions based on scientific theory, discoveries, skills and logical sequences or stages for making patient-focused decisions (Sudarti, 2010).

How midwives deal with climaterium problems in the community. As stated that only about $25 \%$ of women complain that there is a decrease in body estrogen and require additional hormones as a substitute. Hormone substitution without close supervision is dangerous, because midwives can take steps such as doing KIEM so that women with menopausal complaints can go to the puskesmas doctor; midwives consult with puskesmas doctors or specialist doctors; after treatment, midwives can continue monitoring; the midwife can refer the patient to the hospital

\section{Conclusion}

Climacterium is a period that begins at the end of the reproductive stage, ends at the beginning of the senium and occurs in women aged 40-65 years. This period was marked by various kinds of complaints. Climacterium is not a pathological condition, but a normal transitional period that lasts several years before and after menopause. This is because the ovaries become old, so the hormone estrogen decreases and gonadotropin hormone increases. Menopause is physiological for every woman. Postmenopausal women will experience several complaints both physically and psychologically. The symptoms experienced by women before menopause cause the mother to be unprepared about physical and psychological changes. To reduce this, women must prepare themselves both physically and psychologically for menopause. Women who are going through menopause go through the first stages including premenopause, perimenopause, menopause, and postmenopause, and menopause generally occurs in women aged 45-50 years.

\section{References}

Berterö, C. (2003). What do women think about menopause? A qualitative study of women's expectations, apprehensions and knowledge about the climacteric period. International Nursing Review, 50(2), 109-118.

Berterö, C. (2003). What do women think about menopause? A qualitative study of women's expectations, apprehensions and knowledge about the climacteric period. International Nursing Review, 50(2), 109-118.

Eagleson, C. A., Gingrich, M. B., Pastor, C. L., Arora, T. K., Burt, C. M., Evans, W. S., \& Marshall, J. C. (2000). Polycystic ovarian syndrome: evidence that flutamide restores sensitivity of the gonadotropin-releasing hormone pulse generator to inhibition by estradiol and progesterone. The Journal of Clinical Endocrinology \& Metabolism, 85(11), 4047-4052.

Fanfulla, F., Camera, A., Fulgoni, P., Chiovato, L., \& Nappi, R. E. (2013). Sexual dysfunction in obese women: does obstructive sleep apnea play a role?. Sleep medicine, 14(3), 252256.

Graziottin, A., \& Leiblum, S. R. (2005). Biological and psychosocial pathophysiology of female sexual dysfunction during the menopausal transition. The Journal of Sexual Medicine, 2, 133-145.

Hale, G. E., \& Burger, H. G. (2009). Hormonal changes and biomarkers in late reproductive age, menopausal transition and menopause. Best practice \& research Clinical obstetrics \& gynaecology, 23(1), 7-23. 
Levine, K. B., Williams, R. E., \& Hartmann, K. E. (2008). Vulvovaginal atrophy is strongly associated with female sexual dysfunction among sexually active postmenopausal women. Menopause, 15(4), 661-666.

Monteleone, P., Mascagni, G., Giannini, A., Genazzani, A. R., \& Simoncini, T. (2018). Symptoms of menopause-global prevalence, physiology and implications. Nature Reviews Endocrinology, 14(4), 199.

Salvatore, S., Nappi, R. E., Parma, M., Chionna, R., Lagona, F., Zerbinati, N., ... \& Leone Roberti Maggiore, U. (2015). Sexual function after fractional microablative CO2 laser in women with vulvovaginal atrophy. Climacteric, 18(2), 219-225.

Thurston, R. C., Johnson, B. D., Shufelt, C. L., Braunstein, G. D., Berga, S. L., Stanczyk, F. Z., ... \& Merz, C. N. B. (2017). Menopausal symptoms and cardiovascular disease mortality in the Women's Ischemia Syndrome Evaluation (WISE). Menopause (New York, NY), 24(2), 126. 